\title{
In-Flight Suppression of a De-Stabilized F/A-18 Structural Mode Using the Space Launch System Adaptive Augmenting Control System
}

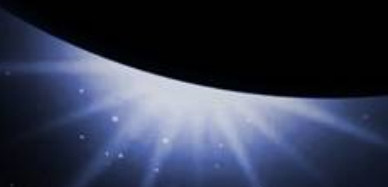

John Wall Dynamic Concepts, Inc. (Jacobs ESSSA Group)

Tannen VanZwieten, Ph.D. NASA Engineering Safety Center

Eric Gilligan NASA Marshall Space Flight Center

Chris Miller and Curtis Hanson NASA Armstrong Flight Research Center 


\section{Introduction}

- Adaptive Augmenting Control (AAC) has been developed for NASA's Space Launch System (SLS) family of launch vehicles and implemented as a baseline part of its flight control system (FCS) [3]

- To raise the technical readiness level of the SLS AAC algorithm, the Launch Vehicle Adaptive Control (LVAC) flight test program was conducted in which the SLS FCS prototype software was employed to control the pitch axis of Dryden's specially outfitted F/A-18, the Full Scale Advanced Systems Test Bed (FAST) [1]

- This presentation focuses on a set of special test cases which demonstrate the successful mitigation of the unstable coupling of an F/A-18 airframe structural mode with the SLS FCS

- Agenda

- Overview of SLS adaptive control system

- Overview of F/A-18 Launch Vehicle Adaptive Control Flight experiment

- Identification of F/A-18 airframe structural mode

- Development of test cases for mode de-stabilization

- Flight test results demonstrating recovery of unstable airframe with adaptive control
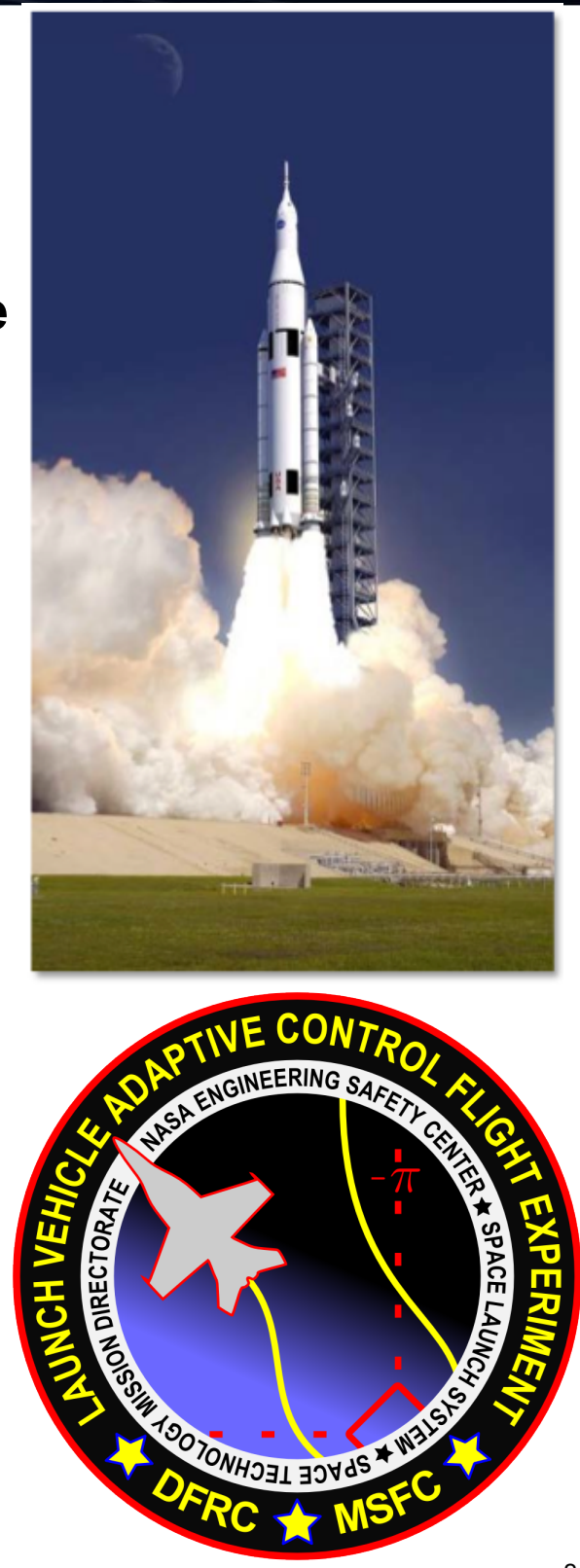


\section{Background: SLS Adaptive Control}

- Adaptive Augmenting Control (AAC) influences a classical, well-tuned PID Flight Control System (FCS) via total loop gain adjustment

AAC Algorithm is comprised of three components, designed to satisfy three corresponding objectives:

1) Do no harm, minimally adapt when not needed (gain returns at 1.0)

- Driven by modified leakage term

$\checkmark 2$ ) Increase command tracking performance (increase the gain)

- Reference model produces error signal

3) Mitigate undesirable parasitic dynamics (decrease the gain)

- Spectral damper indicates undesirable frequency content in control path

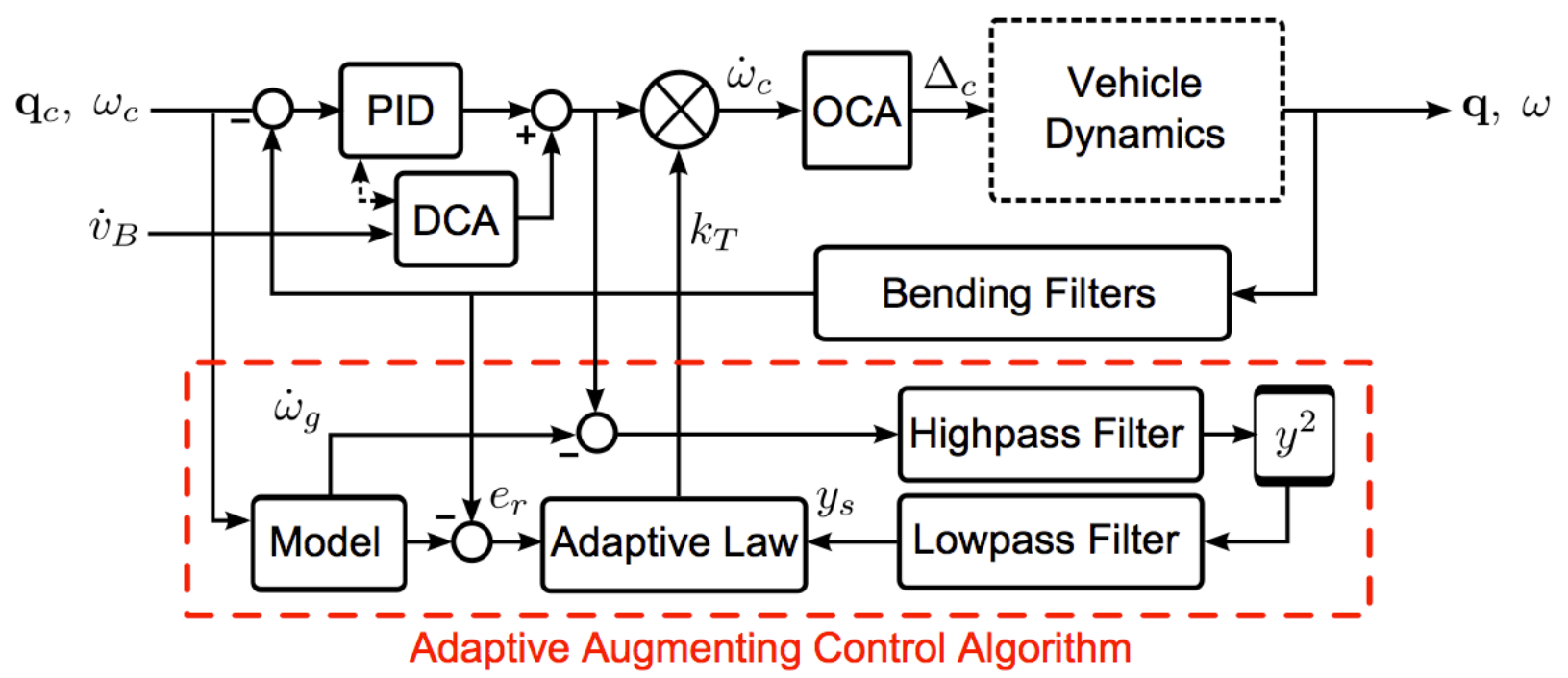




\section{Background: LVAC Flight Test Experiment}

- LVAC experiment was partnership between:

- NASA SLS Program at Marshall Space Flight Center (MSFC)

- NASA Armstrong Flight Research Center (AFRC)

- NASA Engineering and Safety Center (NESC)

- Space Technology Mission Directorate (STMD) Game Changing Development Program (GCDP)

- FIA-18 pitch axis commanded by SLS FCS, SLS Reference models, and F/A-18 Nonlinear Dynamic Inversion (NDI) Control System while on SLS-like trajectory

- F/A-18 flight test cases developed and flown to demonstrate the AAC objectives with SLS-derived nominal, off-nominal, or failure scenarios

- Multiple 60+ minute flights, $100+$ test cases flown

- Special test cases were executed to identify airframe structural dynamics, amplify mode to closed-loop instability, and demonstrate mitigation with SLS AAC
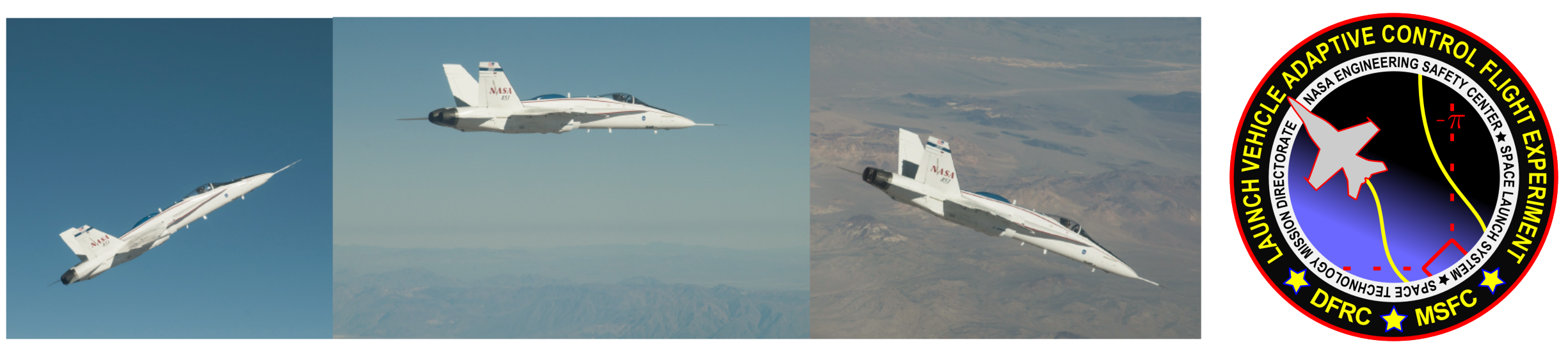


\section{Modal Identification: Excitation Inputs}

- F/A-18 baseline pitch axis control system and the NDI feedback paths during experiment contain $2^{\text {nd }}$ order notch filter at $9.5 \mathrm{~Hz}$ to remove fundamental mode

- No further information was available on modal response

- During first research flight, FLT 140 test card, Test Case (TC) 19 was executed during straight-and-level flight to excite the structural dynamics via a Programmed Test Input (PTI) [5]

- PTI is an angular acceleration cmd consisting of optimized sum-of-sines

- Employed successfully on Ares I-X test flight, and is baselined for first flight test of SLS

$\checkmark$ PTI waveform injected in control path before SLS reference models

- 8-10 Hz, $0.1 \mathrm{~Hz}$ resolution, sample rate $80 \mathrm{~Hz}$

- 20 second waveform, repeated $3 x$ to produce 60 s
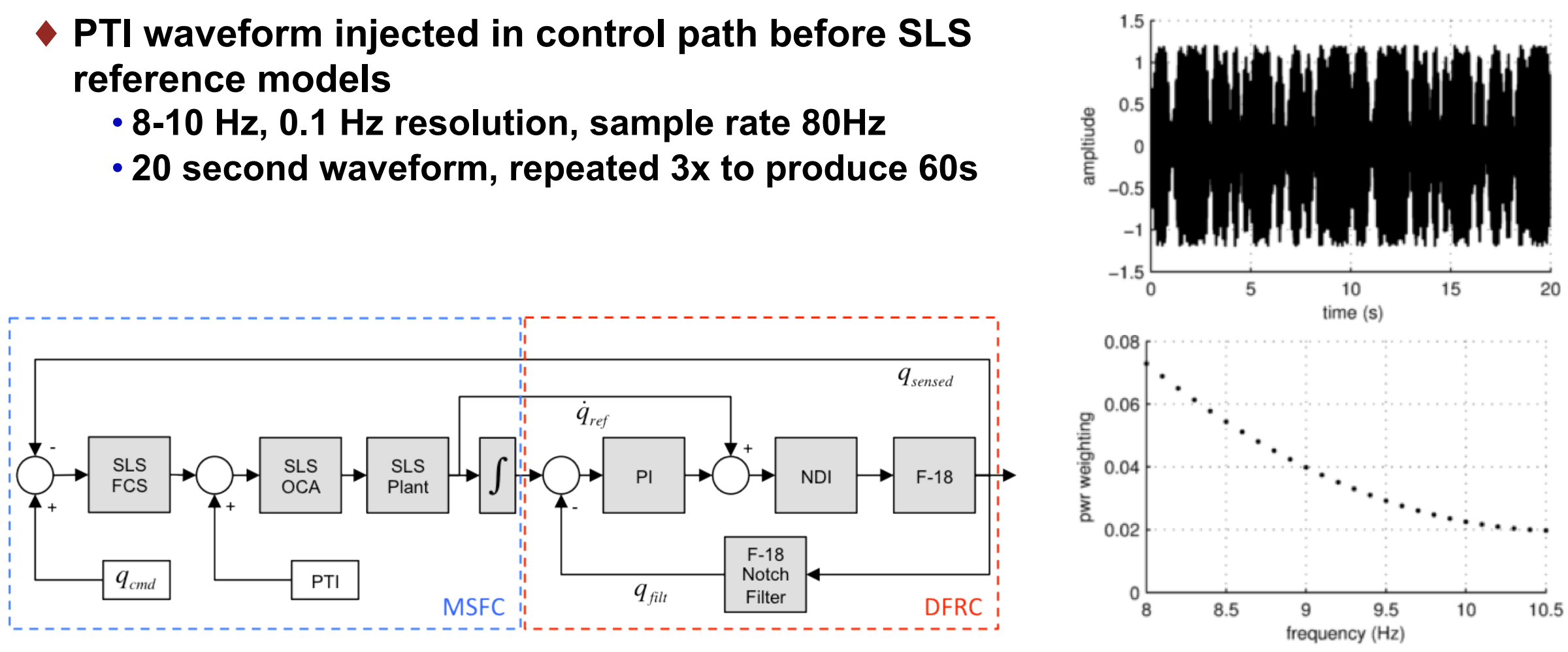


\section{Modal Identification: Flight Test Approach}

$\checkmark$ Modifications to baseline SLS reference model \& controller for structural mode experiment

- SLS actuator limits removed

- SLS parasitic dynamics removed (flex \& slosh), environment model disabled

- NDI control system allocated all control commands to the stabilators to maximize excitation fuselage mode

- Independent variable for SLS FCS fixed at time $=0$

- SLS FCS rate and attitude filters set to unity

- Provided attenuation at $9.5 \mathrm{~Hz}$ comparable to for bulk of SLS-derived test cases

- PTI signal magnitude was configurable via Nose-Wheel-Steering (NWS) button presses in flight

- Built-up approach to ensure safe excitation of potentially high gain mode

- 5x,10x, 15x, 20x, 25x available based on HWIL predictions of actuator rate limiting

- $15 x$, then $20 x$, and then $25 x$ cases flown

- Identification test card (TC 19) engaged during straight and level flight

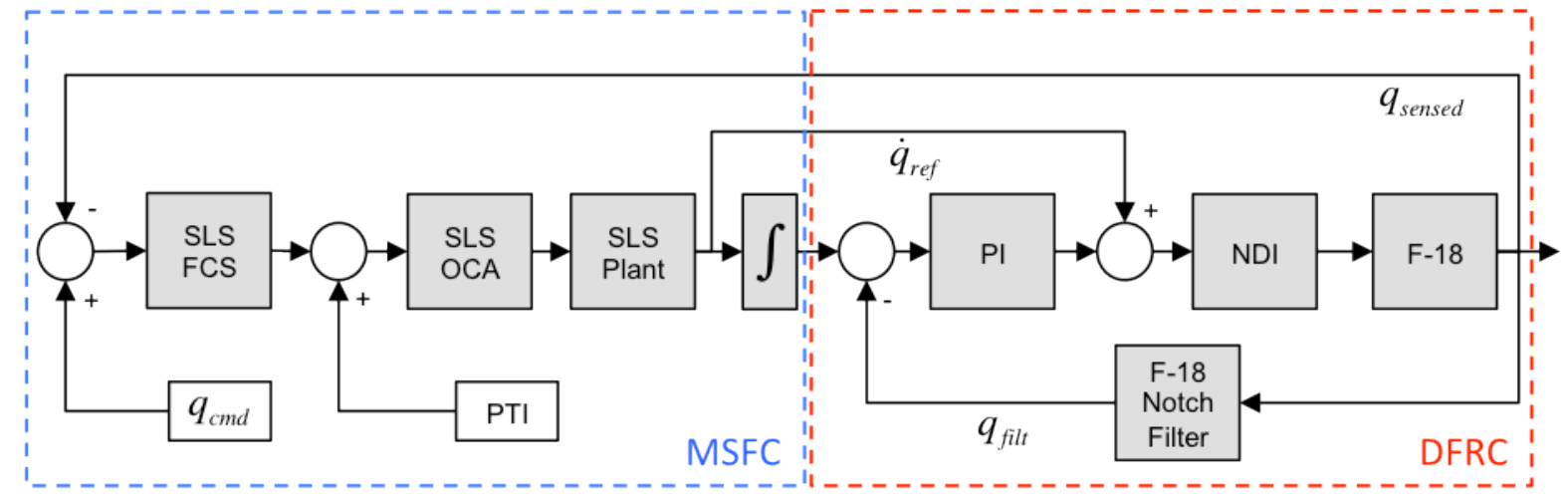

\begin{tabular}{|cc|}
\hline NWS & PTI Gain \\
\hline 0 & 5 \\
1 & 10 \\
2 & 15 \\
3 & 20 \\
4 & 25 \\
\hline
\end{tabular}




\section{Modal Identification: Post Flight Model Fit}

- Keeping the assumed rigid body model parameters constant, numerical optimization was used to derive the $2^{\text {nd }}$ order structural dynamic model coefficients to best fit FFT of flight data from NDI command to sensed pitch rate

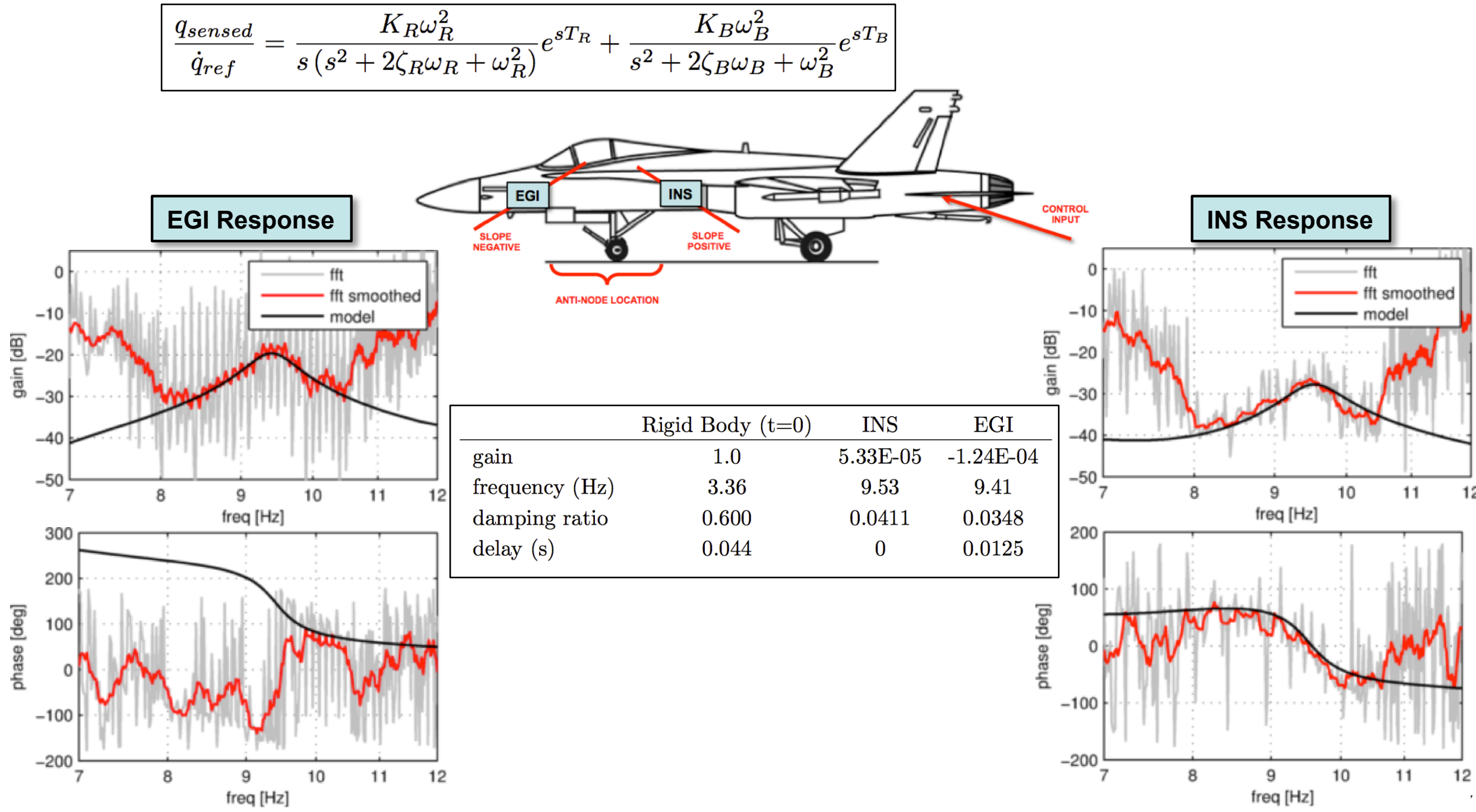




\section{Amplification Test Case Design}

Given model fit for each of the available pitch rate sensors, test cases were developed to intentionally de-stabilize the airframe mode in the control path

- Additional modifications were made to flight software:

- SLS OCA and plant bypassed in SLS reference model by connecting SLS flight control system angular acceleration command directly to F-18 NDI control system

- PTI maneuver time-cropped and gained down to induce a small excitation for a short, 2sec burst at $5 \mathrm{sec}$ after the experiment engaged

- Gain, delay, and bandpass filter applied to SLS FCS output

- Gain configurable via card number

- TC 20: INS feedback test cards

- TC 22: EGI feedback test cards

- NWS configurable frame delay

Bandpass filter designed for each TC 20 \& TC 22

\begin{tabular}{|ccc|}
\hline Test Case & Gain & Card Number \\
\hline 20 & $-3 \mathrm{~dB}$ & 43 \\
& $0 \mathrm{~dB}$ & 44 \\
& $+3 \mathrm{~dB}$ & 45 \\
& $+6 \mathrm{~dB}$ & 46 \\
22 & $-3 \mathrm{~dB}$ & 47 \\
& $0 \mathrm{~dB}$ & 48 \\
& $+3 \mathrm{~dB}$ & 49 \\
& $+6 \mathrm{~dB}$ & 50 \\
\hline
\end{tabular}

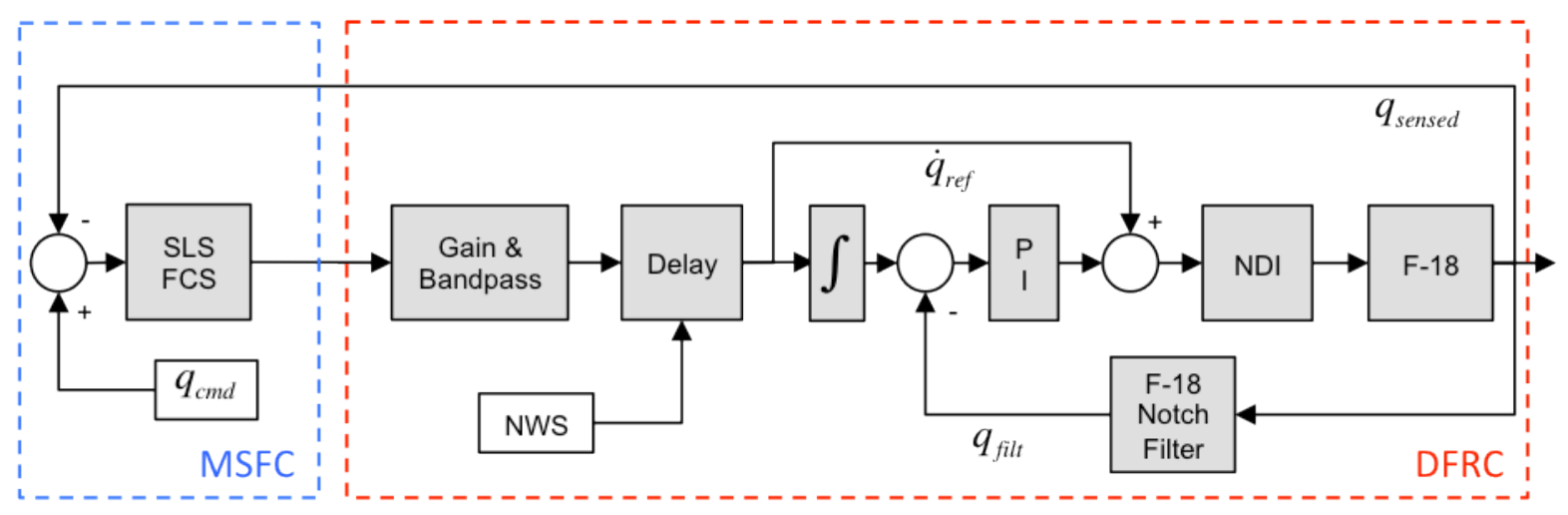

\begin{tabular}{|ccc|}
\hline NWS & Frame Delay & Phase Delay at $9.5 \mathrm{~Hz}$ \\
\hline 0 & $0(0.0000 \mathrm{~s})$ & $00.00 \mathrm{deg}$ \\
1 & $1(0.0125 \mathrm{~s})$ & $42.75 \mathrm{deg}$ \\
2 & $2(0.0250 \mathrm{~s})$ & $85.50 \mathrm{deg}$ \\
3 & $3(0.0375 \mathrm{~s})$ & $128.25 \mathrm{deg}$ \\
4 & $4(0.0500 \mathrm{~s})$ & $171.00 \mathrm{deg}$ \\
\hline
\end{tabular}




\section{Destabilization Filter Design}

De-stabilization filter design took a multi-step approach

- Desire was to place filter which would place mode at $+0 \mathrm{~dB}$ at multiple of $180 \mathrm{deg}$ phase

- In flight, gain \& phase adjusted about nominal point to "find" instability

- Inspection of the open loop system with unity gain \& unity filter determined the level of amplification and amount of phase required to de-stabilized

- Open loop taken at the angular acceleration command output from the SLS FCS

Open loop response with INS feedback shows

- $-29 \mathrm{~dB}$ gain \& 33.03 phase

- Filter/Phase/Gain combination required to add:

$-+29 \mathrm{~dB}$ of gain and 147 deg phase
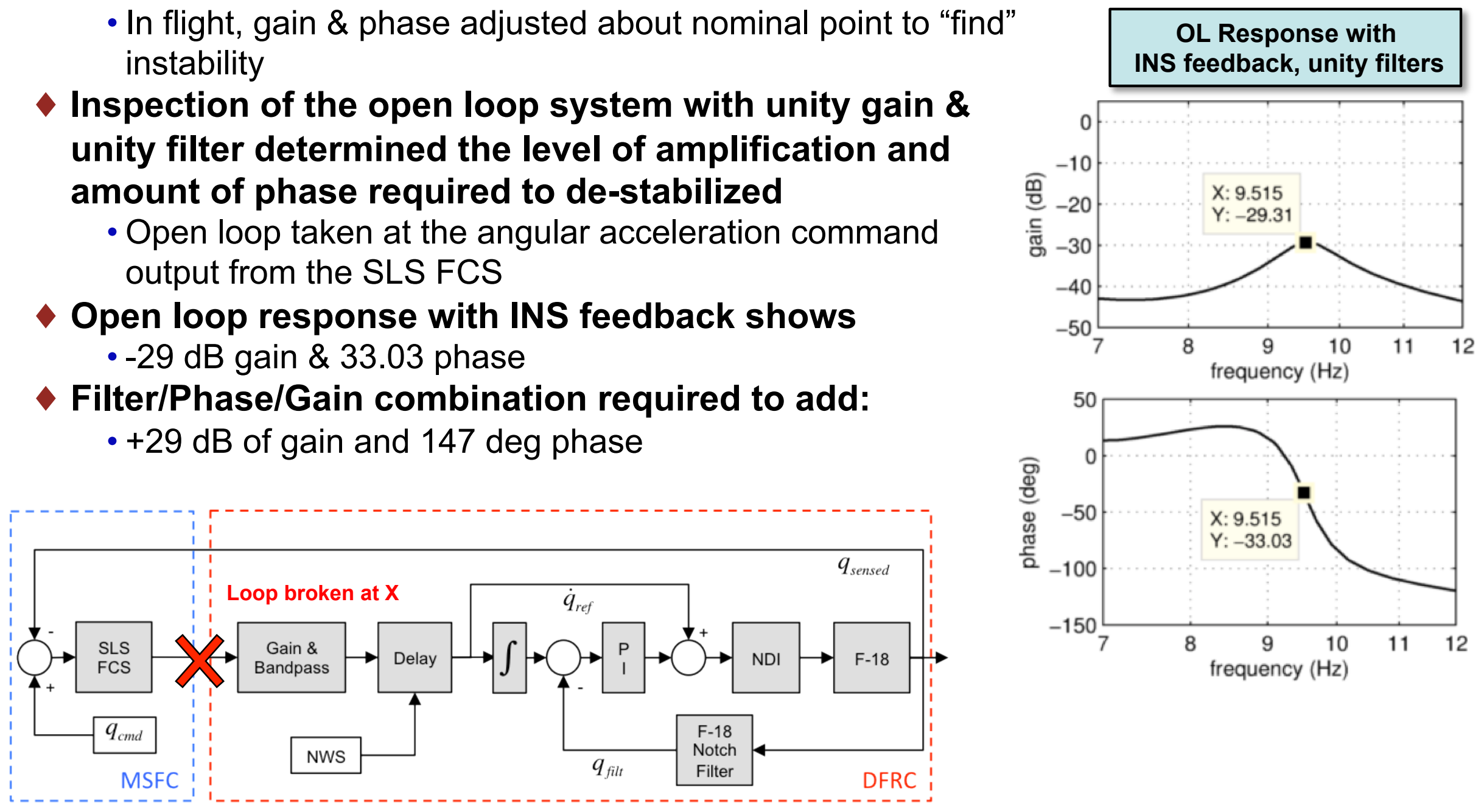


\section{De-stabilization Fiter Results: INS Feedback}

- Using SLS optimal filter design tool [6], coefficients of $8^{\text {th }}$ order filter were solved to produce

- $29 \mathrm{~dB}$ gain

- 85.5 deg phase lag

- Additional phase lag achieved by NWS presses, configuring 2 additional frame delays

-2-80 Hz frames $=108 \mathrm{deg}$ lag

- Selecting $+3 \mathrm{~dB}$ test card produces sufficient gain at 180 deg phase

$\checkmark$ Inspection of the resulting OL system with filter confirms unstable mode

$\checkmark \sim 4 \%$ damped mode provides some phase-error tolerance
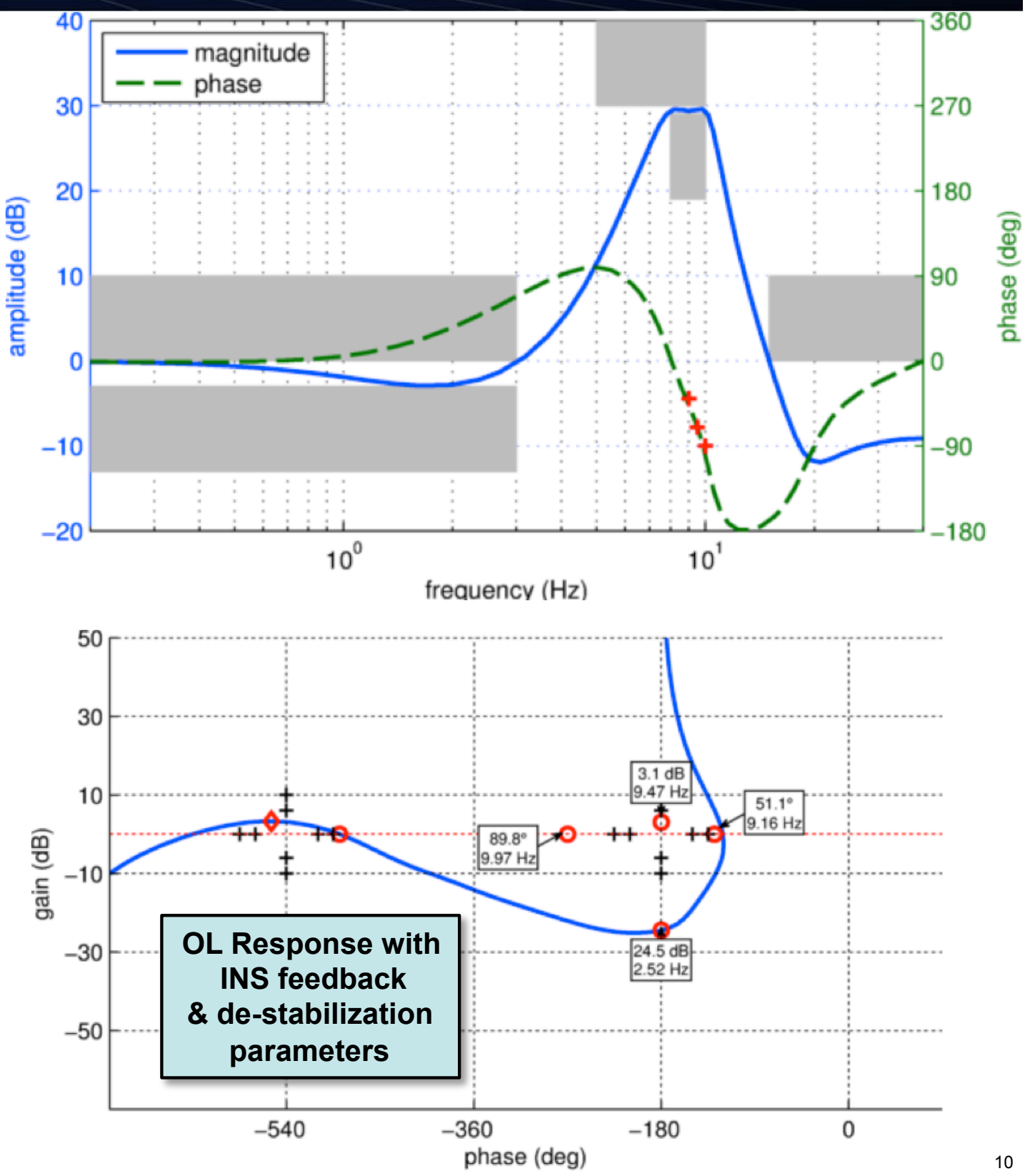


\section{Summary of Filter Design: EGI Results}
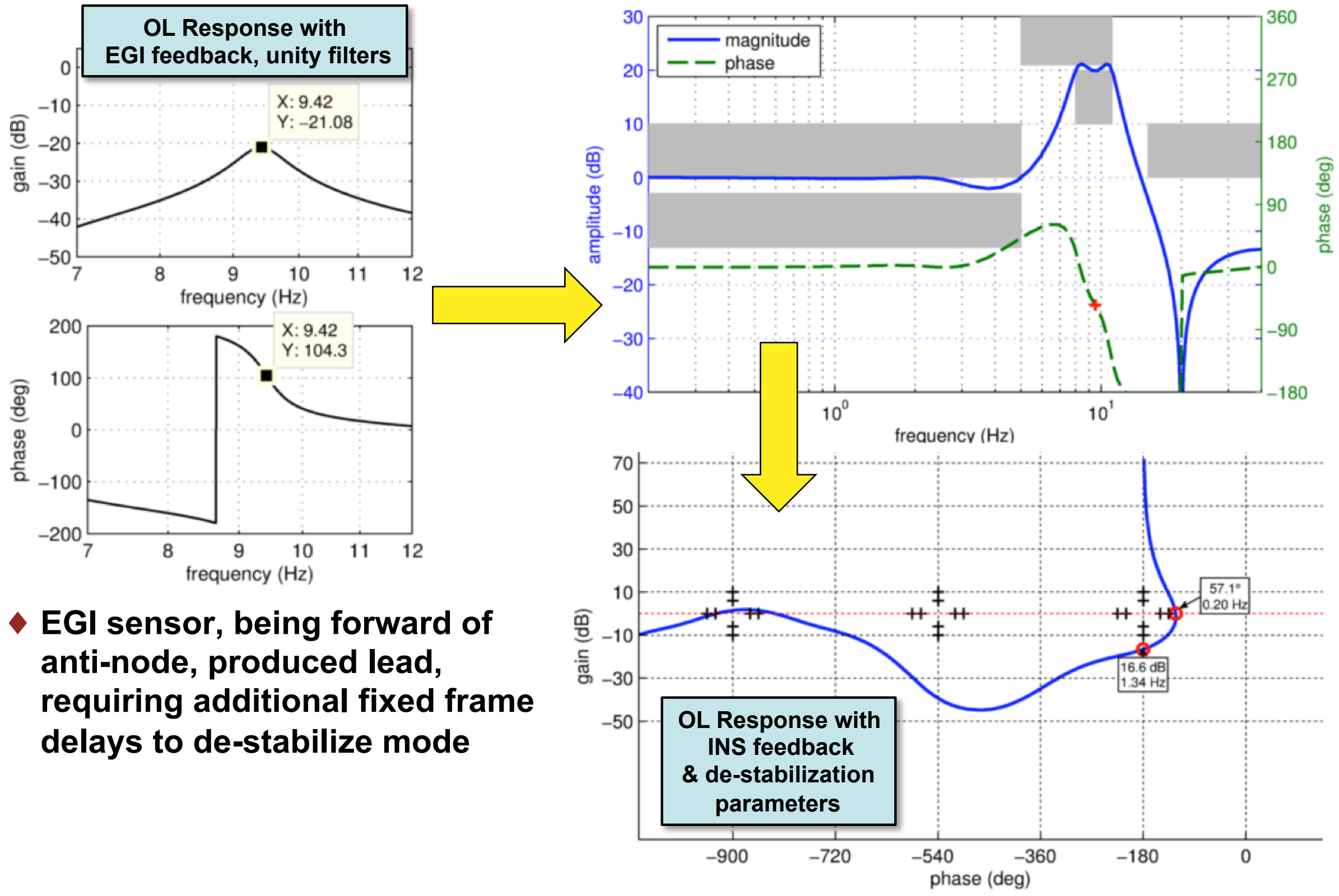

EGI sensor, being forward of anti-node, produced lead, requiring additional fixed frame delays to de-stabilize mode 


\section{Pre-Flight Predictions of De-stabilized Mode: INS}

Results of pre-flight simulation demonstrate expected results in flight

With AAC off, mode will grown unbounded to instability

- Actual flight is expected to saturate actuator rate capability and limit cycle

$\checkmark$ With AAC on, system response remains bounded as a result of the total loop gain adaptation

- Total loop gain exhibits stable oscillatory behavior
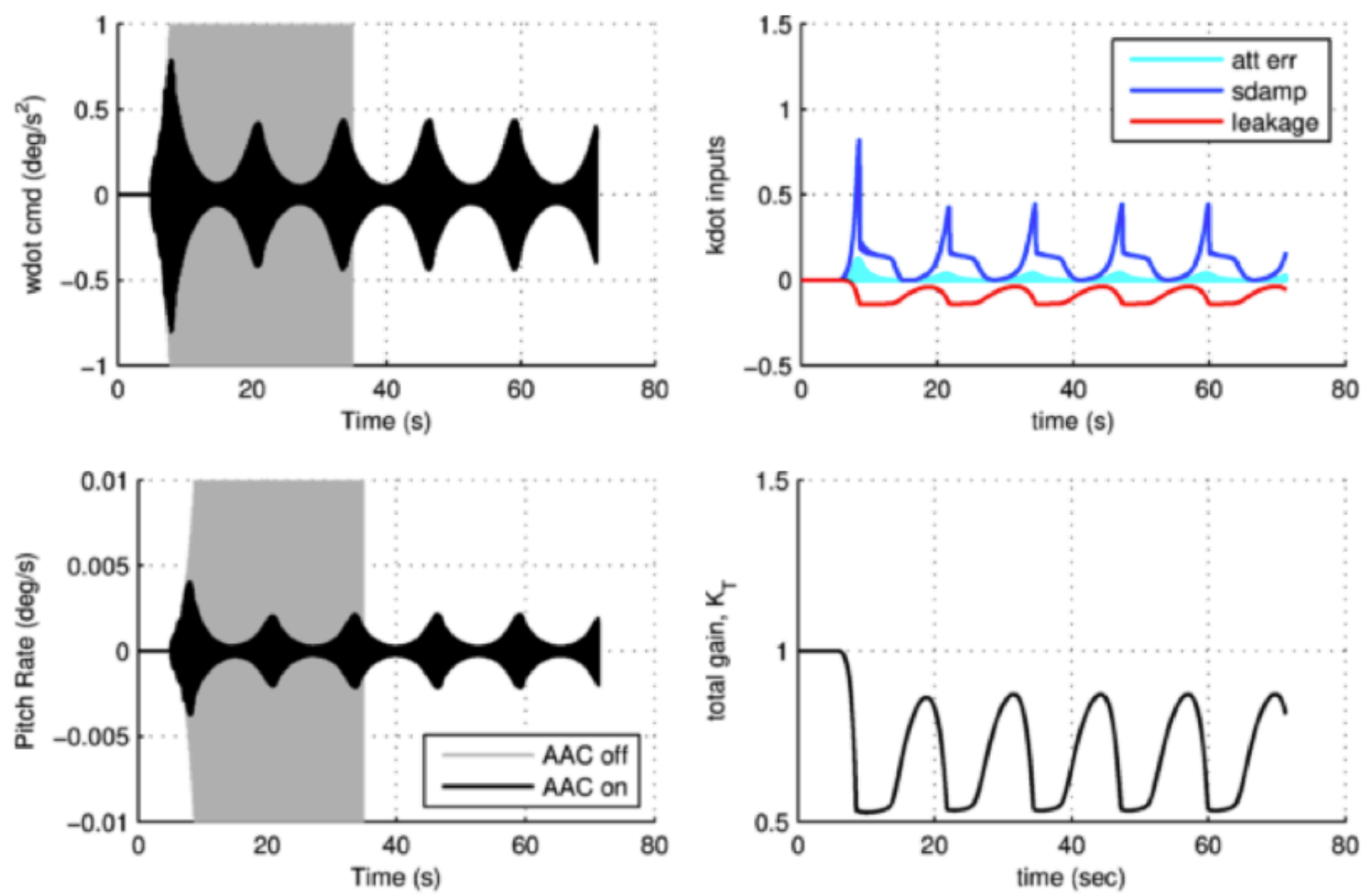
- During the first test flight (Flight 143) in which the de-stabilization cases were tested, the telemetered data confirmed the expected results, with the nominal settings: $+3 \mathrm{~dB}$ gain, 2 frame delays

AAC off case shows the expected growth in the control command to the bounded limit cycle resulting from the rate capability of the stabilator actuators

- AAC on case demonstrates suppression of the instability with a continuously modulating gain behavior similar to pre-flight predictions
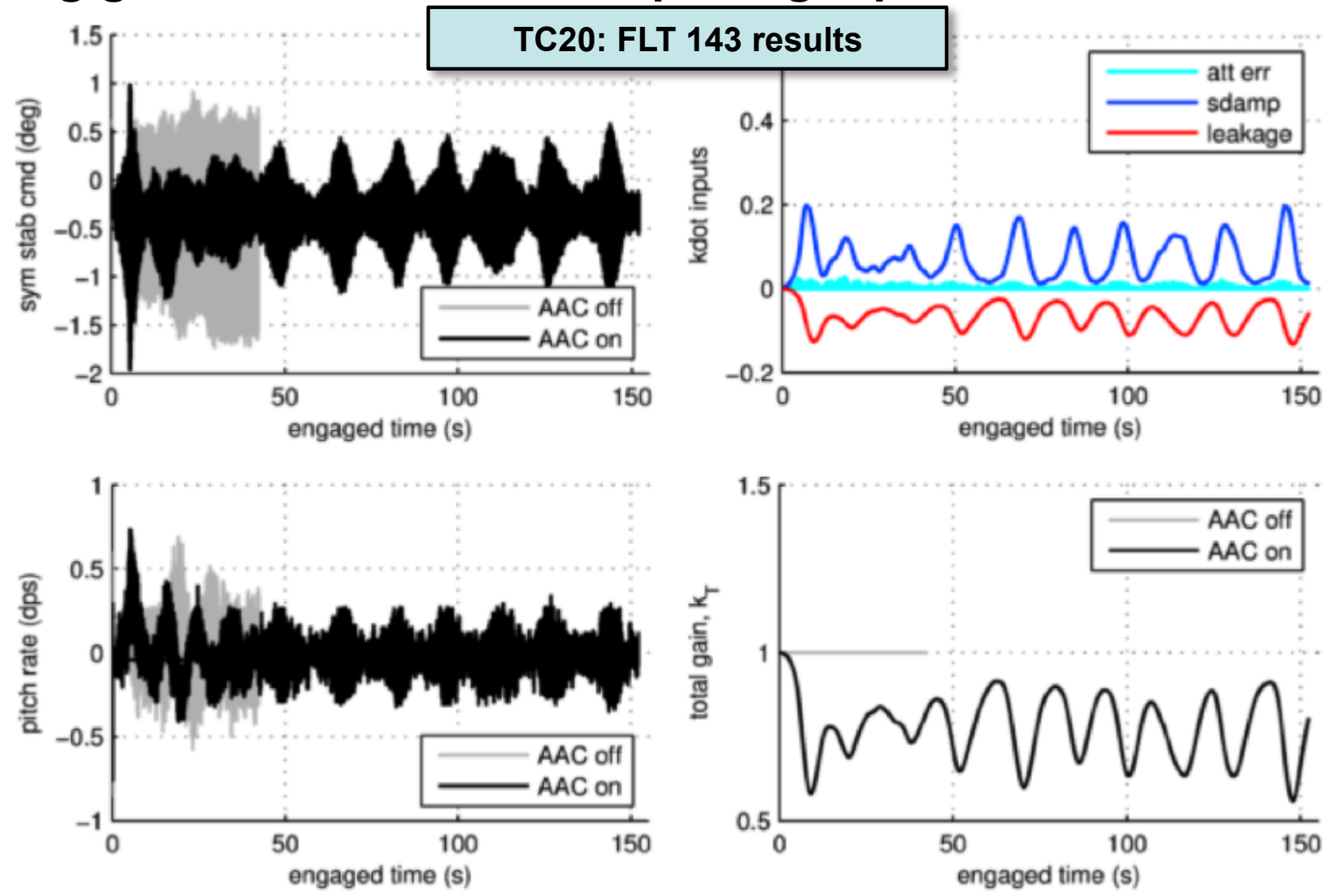


\section{INS case TC20 Flight 144 \& Flight Conditions}

While the first flight of TC20 in FLT 143 showed response on par with pre-flight predictions, subsequent flight, FLT 144, showed similar but reduced response

- Fuel conditions are likely cause of difference

- FLT 143: close match; fuel level was near conditions during ID test FLT 140

- FLT 144 shows less severe instability, fuel level significantly different

\section{- $A A C$ response is proportional to severity}
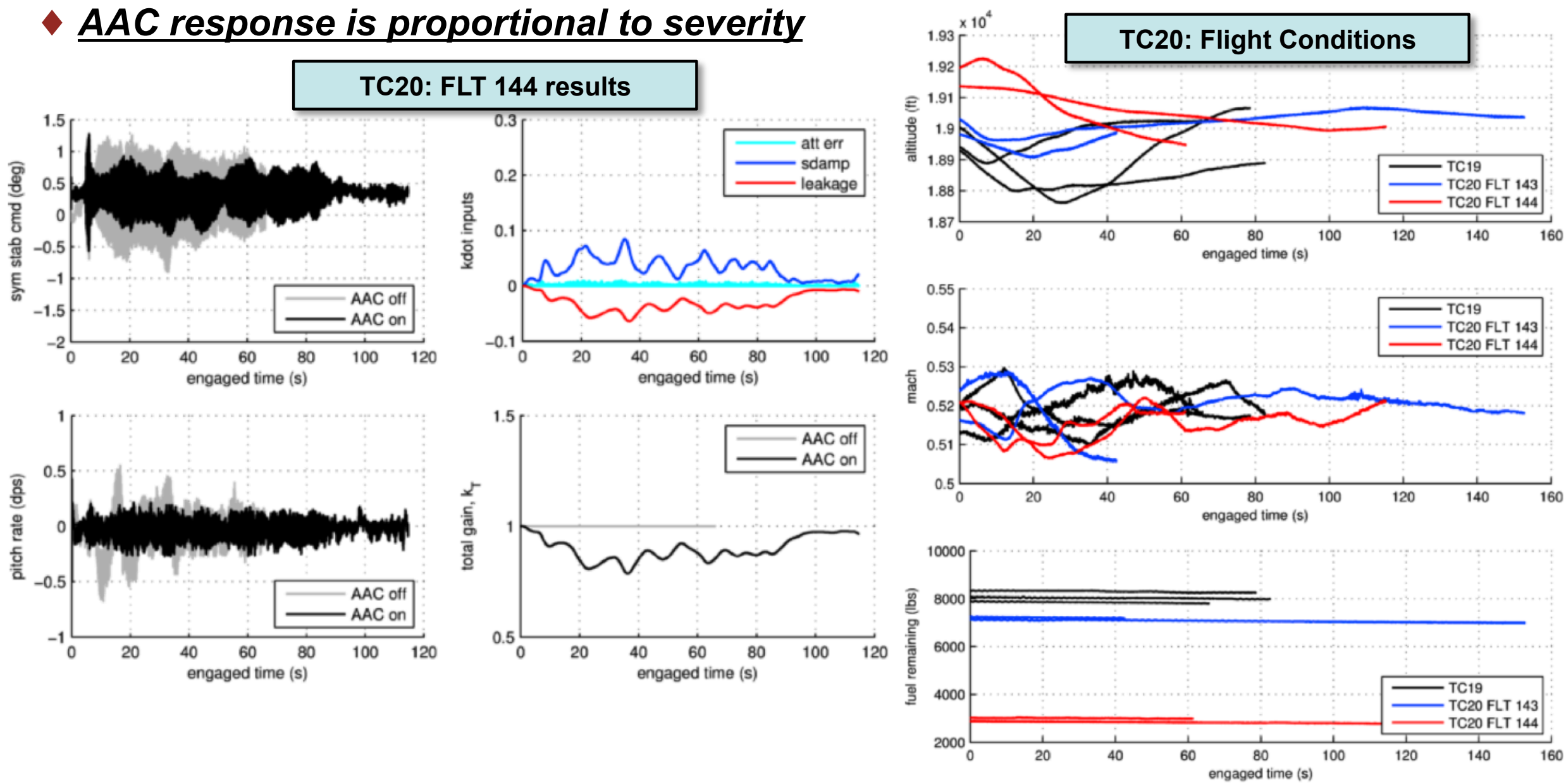


\section{Test Case Conditions: EGI feedback}

While FLT 143 of TC 20 (INS feedback) was an immediate success, the TC22 (EGI feedback) case required a few more iterations in flight until an unstable mode was observed

- Mismatch in part due to uncertainty in EGI phasing (avionics delay uncertainty)

- Instability was observed only at half-full fuel condition
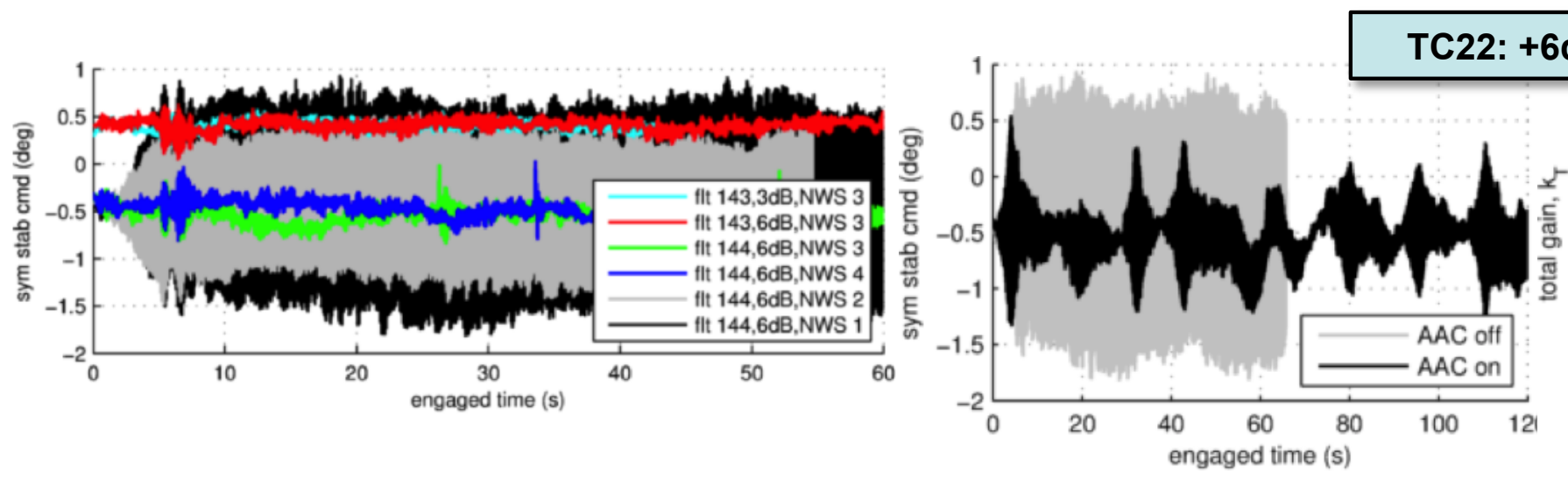

TC22: +6dB, NWS 1
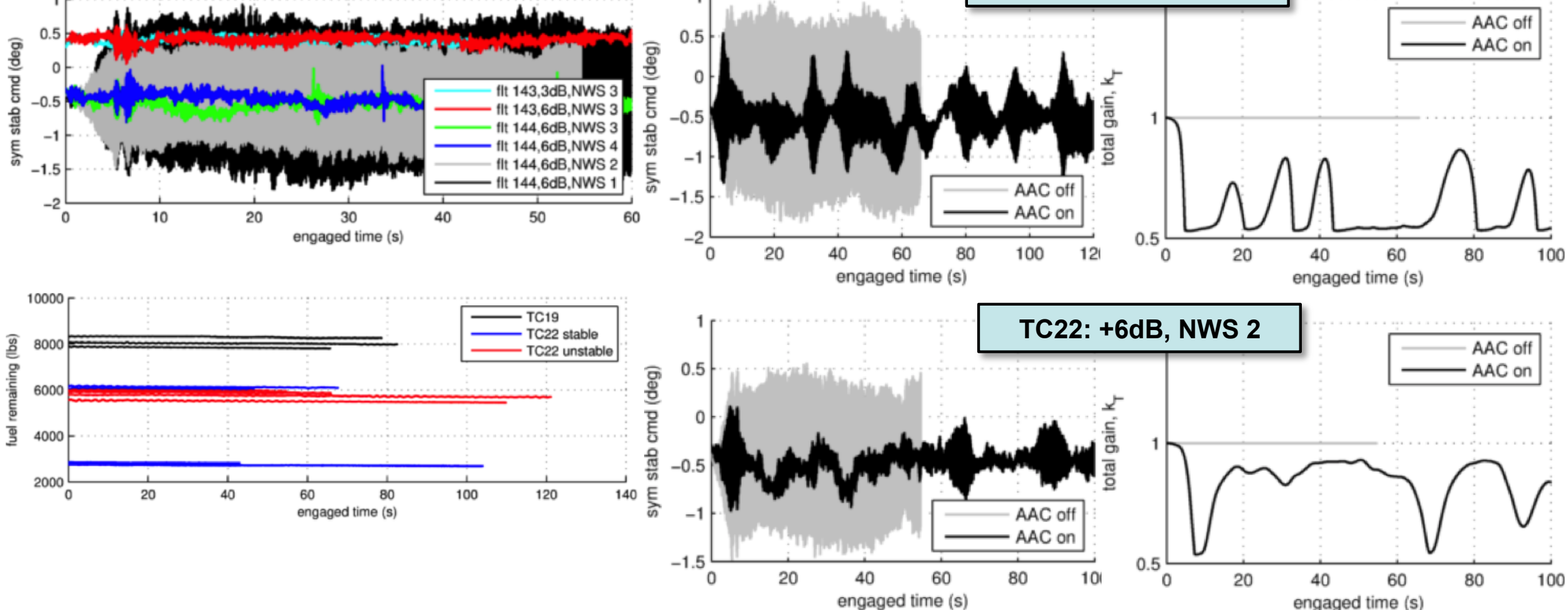

engaged time (s)

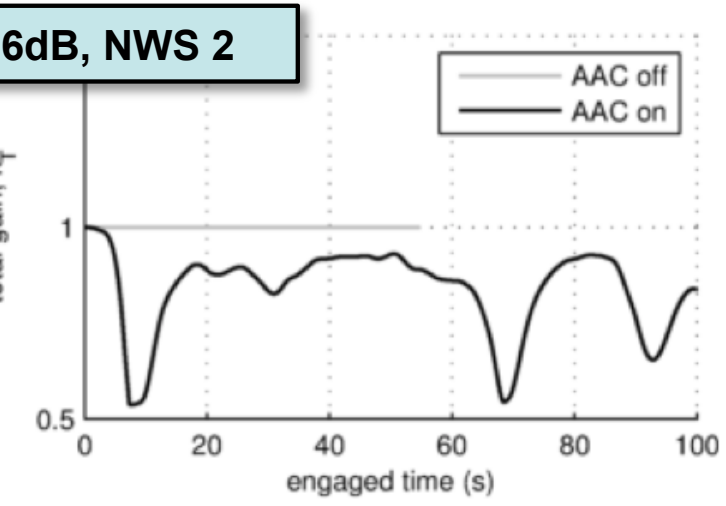

$\checkmark \underline{A A C}$ performs as expected, proportional to severity of instability 


\section{Concluding Remarks}

Test cases were flown during the LVAC experiment which identified the first fuselage mode of the FAST F/A-18 platform

De-stabilizing filters, gains, and delays were placed in control path and produced an unstable control-structure interaction for each of the two available pitch rate sensors

- The SLS Adaptive Augmenting Control algorithm demonstrated the ability to suppress the unstable dynamics and show similar response to simulated results

- Fuel conditions were found to be the cause of greatest discrepancy in structural dynamics predictions

AAC performed proportional to level of instability 
(1) VanZwieten, T., Gilligan, E., Wall, J., Orr, J., Miller, C., and Hanson, C., "Adaptive Augmenting Control Flight Characterization Experiment on an F/A-18," AAS Guidance, Navigation, and Control Conference, Breckenridge, CO, 2014.

(2) J. Orr and T. VanZwieten, "Robust, Practical Adaptive Control for Launch Vehicles," AIAA Guidance, Navigation, and Control Conference, Minneapolis, MN, August 2012.

(3) Wall, J., Orr, J., and VanZwieten, T., "Space Launch System Implementation of Adaptive Augmenting Control," AAS Guidance, Navigation, and Control Conference, Breckenridge, CO, 2014.

(4) Miller, C., "Nonlinear Dynamic Inversion Baseline Control Law: Architecture and Performance Predictions," AIAA Guidance, Navigation, and Control Conference, Portland, OR, 2011.

(5) Morelli, E. A., "Multiple Input Design for Real-time Parameter Estimation in the Frequency Domain," Paper REG-360, 13th IFAC Symposium on System Identification, Rotterdam, The Netherlands, August 2003.

(6) Orr, J., "Optimal Recursive Digital Filters for Active Bending Stabilization," AAS Guidance, Navigation, and Control Conference, Breckenridge, CO, 2013. 